OPEN ACCESS

Edited by:

Wenli Cai,

Massachusetts General Hospital and Harvard Medical School, United States

Reviewed by:

Zhongxiang Ding,

Zhejiang University, China

Guobin Hong,

The Fifth Affiliated Hospital of Sun

Yat-sen University, China

*Correspondence:

Maria Luisa Belli

maria.belli@irst.emr.it

${ }^{t}$ These authors have contributed equally to this work and share first authorship

Specialty section: This article was submitted to Cancer Imaging and Image-directed Interventions, a section of the journal Frontiers in Oncology

Received: 31 August 2020 Accepted: 31 May 2021 Published: 24 June 2021

Citation:

Fantini L, Belli ML, Azzali I, Loi E, Bettinelli A, Feliciani G, Mezzenga E, Fedeli A, Asioli S,

Paganelli G, Sarnelli A and Matteucci $F$ (2021) Exploratory

Analysis of ${ }^{18} \mathrm{~F}-3^{\prime}$-deoxy-3'fluorothymidine $\left({ }^{18} \mathrm{~F}-\mathrm{FL}\right.$ T) PET/CT-

Based Radiomics for the Early

Evaluation of Response to Neoadjuvant Chemotherapy in

Patients With Locally

Advanced Breast Cancer.

Front. Oncol. 11:601053. doi: 10.3389/fonc.2021.601053

\section{Exploratory Analysis of ${ }^{18} \mathrm{~F}-3^{\prime}$-deoxy- 3'-fluorothymidine ( $\left.{ }^{18} \mathrm{~F}-\mathrm{FLT}\right)$ PET/CT- Based Radiomics for the Early Evaluation of Response to Neoadjuvant Chemotherapy in Patients With Locally Advanced Breast Cancer}

\author{
Lorenzo Fantini ${ }^{1 \dagger}$, Maria Luisa Belli ${ }^{2 *+}$, Irene Azzali ${ }^{3}$, Emiliano Loi $^{2}$, Andrea Bettinelli ${ }^{4}$, \\ Giacomo Feliciani ${ }^{2}$, Emilio Mezzenga ${ }^{2}$, Anna Fedeli ${ }^{5}$, Silvia Asioli ${ }^{6}$, Giovanni Paganelli ${ }^{1}$, \\ Anna Sarnelli ${ }^{2}$ and Federica Matteucci ${ }^{1}$

\begin{abstract}
${ }^{1}$ Nuclear Medicine Unit, IRCCS Istituto Romagnolo per lo Studio dei Tumori (IRST) "Dino Amadori," Meldola, Italy, ${ }^{2}$ Medical Physics Unit, IRCCS Istituto Romagnolo per lo Studio dei Tumori (IRST) "Dino Amadori," Meldola, Italy, ${ }^{3}$ Biostatistics and Clinical Trials Unit, IRCCS Istituto Romagnolo per lo Studio dei Tumori (IRST) "Dino Amadori," Meldola, Italy, ${ }^{4}$ Medical Physics Department, Veneto Institute of Oncology IOV - IRCCS, Padua, Italy, ${ }^{5}$ Department of Medical Oncology, IRCCS Istituto Romagnolo per lo Studio dei Tumori (IRST) "Dino Amadori," Meldola, Italy, ${ }^{6}$ Pathology Unit, Morgagni-Pierantoni Hospital, Forli, Italy
\end{abstract}

Purpose: The objective of this study was to evaluate a set of radiomics-based advanced textural features extracted from ${ }^{18} \mathrm{~F}$-FLT-PET/CT images to predict tumor response to neoadjuvant chemotherapy (NCT) in patients with locally advanced breast cancer (BC).

Materials and Methods: Patients with operable (T2-T3, NO-N2, MO) or locally advanced (T4, NO-N2, MO) BC were enrolled. All patients underwent chemotherapy (six cycles every 3 weeks). Surgery was performed within 4 weeks of the end of NCT. The MD Anderson Residual Cancer Burden calculator was used to evaluate the pathological response. ${ }^{18} \mathrm{~F}$ FLT-PET/CT was performed 2 weeks before the start of NCT and approximately 3 weeks after the first cycle. The evaluation of PET response was based on EORTC criteria. Standard uptake value (SUV) statistics (SUV max $\left., S U V_{\text {peak }}, S U V_{\text {mean }}\right)$, together with 148 textural features, were extracted from each lesion. Indices that are robust against contour variability (ICC test) were used as independent variables to logistically model tumor response. LASSO analysis was used for variable selection.

Results: Twenty patients were included in the study. Lesions from 15 patients were evaluable and analyzed: 9 with pathological complete response $(\mathrm{pCR})$ and 6 with pathological partial response (pPR). Concordance between PET response and histological examination was found in 13/15 patients. LASSO logistic modelling identified a combination of SUV $V_{\max }$ and the textural feature index IVH_VolumelntFract_90 as the 
most useful to classify PET response, and a combination of PET response, ID range, and ID_Coefficient of Variation as the most useful to classify pathological response.

Conclusions: Our study suggests the potential usefulness of FLT-PET for early monitoring of response to NCT. A model based on PET radiomic characteristics could have good discriminatory capacity of early response before the end of treatment.

\section{Keywords: radiomics, fluorothymidine positron emission tomography scan, breast cancer, early response, neoadjuvant chemotherapy}

\section{INTRODUCTION}

Neoadjuvant chemotherapy (NCT) followed by surgery represents the standard strategy in the treatment of locally advanced breast cancer, obtaining an objective response rate of around $70 \%$ and a complete pathological response rate of up to $30 \%(1-3)$.

It is well known that the pathological response to NCT represents an important prognostic factor in this setting (4-6). However, the evaluation of the response is mainly based on the histopathological findings of the surgical sample. There is thus a clear need for an ongoing evaluation of chemotherapy to early differentiate between responders and non-responders, which would enable the latter to be offered alternative therapies, reduce the risk of chemotherapy-related toxicity, and lower costs for the National Health Service.

At present, there is no unanimous agreement about the best imaging method for the early assessment of response to therapy. Morphology-based imaging methods are generally used and interpreted by Response Evaluation Criteria in Solid Tumors criteria (RECIST v1.1) (7). However, given that morphological changes may arise late in the course of treatment, RECIST v1.1 criteria may not be able to identify response at an earlier stage. Existing evidence of changes in tumor morphology, likely preceded by changes in metabolism, has led to the use of functional imaging methods such as positron emission tomography (PET) for assessing early response to therapy.

Currently, ${ }^{18} \mathrm{~F}$-labeled fluorodeoxyglucose (FDG) is the most widely used method to monitor response to therapy in BC. Although ${ }^{18}$ F-FDG is a sensitive tracer, it is not highly tumorspecific as it also accumulates in activated macrophages and other inflammatory cells, with a consequent increase in potential false-positive responses (8).

In the search for more specific tracers for $\mathrm{BC},{ }^{18} \mathrm{~F}$-labeled fluorothymidine $\left({ }^{18} \mathrm{~F}\right.$-FLT) has been proposed as an indicator of proliferation (9) because thymidine is an analog of pyrimidine, which is incorporated in DNA but not in RNA. Thus, the possibility of quantifying the proliferative activity of the tumor through the use of ${ }^{18} \mathrm{~F}$-FLT PET/CT could represent a potentially useful tool for evaluating the viability of tumor cells during or at the start of treatment.

The most widely used parameter to measure lesion uptake is the standard uptake value (SUV). Semi-quantitative SUV statistics (e.g. $\left.\mathrm{SUV}_{\text {max }}, \mathrm{SUV}_{\text {peak }}, \mathrm{SUV}_{\text {mean }}\right)$ are generally used to measure uptake inside a region. The potential utility of ${ }^{18} \mathrm{~F}$-FLT $\mathrm{PET} / \mathrm{CT}$ images as an early indicator of treatment response, in terms of SUV $\mathrm{Smax}_{\text {matients }}$ in pandergoing NCT was previously demonstrated by Crippa et al. (10).

Although semi-quantitative SUV indices provide overall information on uptake, they are not capable of detecting the presence of non-uniform uptake distribution. However, it is known that tracer uptake within a tumor mass is characterized by significant heterogeneity because of various factors such as metabolism, hypoxia, necrosis, and cell proliferation (11). This heterogeneity appears to correlate with tumor aggressiveness, response to treatment, and prognosis (12).

Radiomics, an approach capable of quantifying the heterogeneity of textures in medical imaging, is an emerging translational research field that may be able to provide more accurate information than the semi-quantitative parameters normally used. The application of radiomic analysis capable of extracting textural features has been used for FDG PET/CT images, with good results (13-16). The availability of textural features before therapy or during the first treatment phases could thus facilitate decision-making in relation to the therapeutic strategy to adopt.

We evaluated a subgroup of patients enrolled in a multicenter phase II trial of liposomal doxorubicin, docetaxel, and trastuzumab in combination with metformin as NCT for HER2-positive BC. The main objective of the present study was to investigate the role of radiomics-based advanced imaging features extracted from ${ }^{18} \mathrm{~F}$-FLT PET/CT images to predict tumor response to NCT in patients with BC.

\section{MATERIALS AND METHODS}

\section{Patients}

We selected 20 patients with operable (T2-T3, N0-N2, M0) or locally advanced (T4, N0-N2, M0) human epidermal growth factor receptor 2-positive (HER2-positive) BC taking part in multicenter phase II trial at our cancer (IRST IRCCS).

All patients gave written consent to participate in the study, which was approved by the Local Research and Ethics Committee (Eudract no. 2014-002602-20; Protocol Code: IRST174.09; ClinicalTrials.gov NCT02488564).

Before surgery, all patients were submitted to chemotherapy with liposome-encapsulated doxorubicin (every 3 weeks, for six cycles) plus docetaxel (every 3 weeks, for six cycles) plus trastuzumab plus metformin $(1,000 \mathrm{mg}$ twice a day per os) (Figure 1A). 
Surgery was performed within 2 to 4 weeks of the end of chemotherapy followed by radiotherapy to the residual breast level (for patients undergoing conservative surgery) or chest wall (T4 tumors).

After surgery, if deemed suitable by the investigator, patients were treated with adjuvant anthracycline/paclitaxel or with the cyclophosphamide, methotrexate, and 5-fluorouracil (CMF) scheme. Herceptin was administered every 3 weeks for 1 year.

\section{Imaging Protocols}

${ }^{18}$ F-FLT was produced by Advanced Accelerator Applications (AAA) with a radiochemical purity and specific activity $>95 \%$ and $>1 \mathrm{Ci} / \mu \mathrm{mol}$, respectively.

All patients underwent a ${ }^{18} \mathrm{~F}$-FLT-PET/CT scan a maximum of 2 weeks before the start of NCT (FLT1, basal) and immediately before the second cycle of chemotherapy (FLT2) (Figure 1B). Patients were weighed before each scan and given an intravenous injection of $3.5 \mathrm{MBq} / \mathrm{kg}$ of FLT (maximum activity $350 \mathrm{MBq}$ ). No dietary restrictions were required before imaging. All patients were scanned in the supine position and were informed of the importance of remaining perfectly still and of maintaining shallow breathing throughout the procedure.

Images were acquired on two different PET/CT scanners. The first scanner was a Discovery LS (GE Healthcare, UK) equipped with 18 rings (92.7 $\mathrm{cm}$ diameter), each containing $672 \mathrm{BGO}$ crystals ( $4 \mathrm{~mm} \times 8 \mathrm{~mm} \times 30 \mathrm{~mm}$ crystal size), $152 \mathrm{~mm}$ axial FOV dimension, combined with a four-slice CT system. Step-andshoot mode was used for whole-body image acquisition. The second scanner was a Biograph mCT Flow 64-4R PET/CT
(Siemens Healthcare, Germany) equipped with four rings (84.2 cm diameter) of 48 detector blocks, each containing $13 \times$ 13 LSO crystals $(4 \mathrm{~mm} \times 4 \mathrm{~mm} \times 20 \mathrm{~mm}$ crystal size), $221 \mathrm{~mm}$ axial FOV dimension, combined with a 64-slice CT system. Continuous table motion mode was used for whole-body image acquisition (17). Both point-spread-function and timeof-light corrections are available on this scanner. Both FLT1 and FLT2 were acquired on the same scanner for each patient.

Image acquisition started $1 \mathrm{~h}$ after intravenous injection of ${ }^{18}$ F-FLT: a CT was performed from the brain to the pelvis immediately before the PET scan, with a multidetector spiral CT scanner (Discovery LS: $3.9 \times 3.9 \mathrm{~mm}^{2}$ pixel dimension, $5 \mathrm{~mm}$ slice thickness, pitch $1.75,120 \mathrm{keV}$ and $20-200$ auto $\mathrm{mA}$; Biograph: $0.98 \times 0.98 \mathrm{~mm}^{2}$ pixel dimension, $3 \mathrm{~mm}$ slice thickness, pitch 1.2, $120 \mathrm{keV}$ and auto $\mathrm{mA} \mathrm{[30-200} \mathrm{mA}$ depending on the patient's total body mass]). Whole-body PET scan was performed, covering an area identical to that covered by the CT. PET data were reconstructed into a $128 \times 128$ matrix (voxel dimension $3.9 \times 3.9$ and $4.1 \times 4.1 \mathrm{~mm}^{2}$, slicethickness 4.25 and $3 \mathrm{~mm}$, for Discovery LS and Biograph mCT Flow scanner, respectively), using the OSEM reconstruction algorithm. Corrections were applied for attenuation, scatter, random coincidences, isotope decay, and dead time. Fused PET and CT images were subsequently generated.

\section{Image Analysis \\ Volume Definition}

All volumes of interest (VOIs) encompassing the lesions were manually contoured on both FLT1 and FLT2 CT images by an
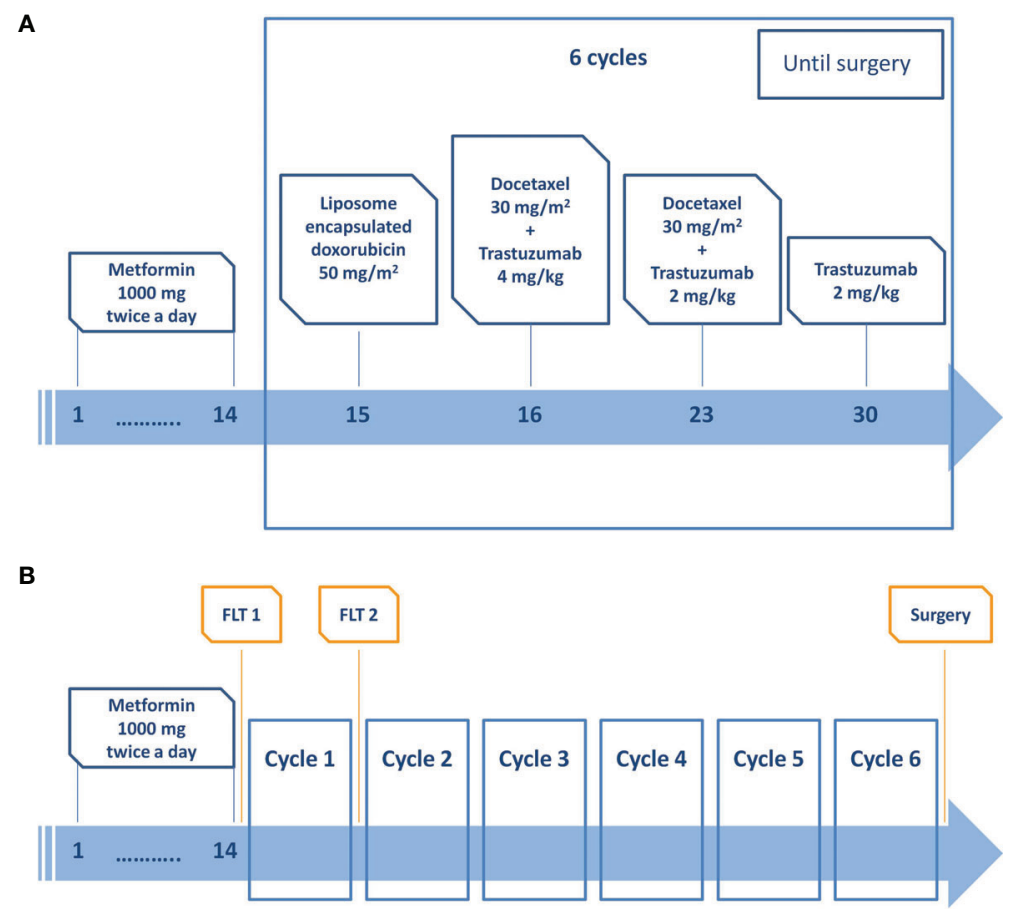

FIGURE 1 | (A) Treatment scheme and timeline. (B) Image acquisition scheme and timeline. 
expert nuclear medicine radiologist (LF) blinded to patient outcome. A second senior nuclear medicine radiologist (FM) supervised each contour. The MimVista software (Mim Software Inc., v6.6, OH, USA) was used for PET/CT image rigid registration and lesion delineation.

In order to test the robustness of textural features against contour variability, four contours (original lesion VOI, expansion of $+1 \mathrm{~mm},+2 \mathrm{~mm}$, contraction of $-1 \mathrm{~mm}$ ) were considered.

PET image and structure set were then extrapolated in DICOM format for textural feature image analysis.

\section{SUV Statistics}

Vendor software was used for SUV statistics calculation. SUV values were derived from the radioactivity concentration in the tissue, the dose of radioactivity administered and the patient's weight. All SUV values were corrected for a patient's body weight. Maximum, mean, and peak SUV values were calculated inside each delineated lesion $\left(\mathrm{SUV}_{\text {max }}, \mathrm{SUV}_{\text {mean }}, \mathrm{SUV}_{\text {peak, }}\right.$ respectively) (Supplementary Materials 1). Each lesion VOI was copied on the contralateral breast, and the corresponding SUV values subtracted for background correction. The percentage variation was then calculated between FLT1 and FLT2 for all SUV statistics.

\section{Texture Analysis}

Feature extraction was performed with the open-source S-IBEX software, implemented in MatLab environment (Math-Works, Boston, MA, USA) (18) and IBSI compliant (19, 20). Voxels were resampled to $1 \times 1 \times 1 \mathrm{~mm}^{3}$ size to take into account the different acquisition parameters of the image sample. A total of 148 features were extracted: 25 first order features (i.e. based on SUV histogram, namely Voxel statistics), 28 morphological features, and 95 second or higher orders (Supplementary Materials 1). Gray level quantization was fixed to 32 bins for second order features and IVH (Intensity Volume Histogram) features.

\section{Response Evaluation Radiological PET Response}

The PET response to therapy was determined according to EORTC 1999 criteria (21) between FLT1 and FLT2.

\section{Pathological Response Evaluation}

At the end of the chemotherapy course, all patients underwent surgery (Table 1). Histopathological analysis was performed and details are reported in Supplementary Materials 2. To evaluate the pathological response based on histopathological findings, we opted for the web MD Anderson Residual Cancer Burden (RCB) calculator $(22,23)$. This software enabled us to identify four different categories of RCB: RCB-0, RCB-I, RCB-II, and RCB-III corresponding to complete pathological response, presence of minimal residual disease (almost complete response), presence of moderate residual disease, and presence of extensive residual disease, respectively. We classified pathological responses as complete (pCR) for both RCB-0 and RCB-I, and partial (pPR) for both RCB-II and RCB-III.

\section{Statistical Analysis}

Continuous data corresponding to SUV statistics and the 148 texture features were considered as percentage changes between FLT1 values and FLT2 values with respect to FLT1: (FLT1-FLT2)/ FLT ${ }^{\star} 100$. This has a threefold purpose: 1$)$ it reflects the reasoning of the clinician who is accustomed to evaluating changes from baseline (i.e. FLT1); 2) it reflects the longitudinal nature of the study; and 3) the use of percentage changes in place of absolute values allows the comparison of results obtained with different scanners.

In order to identify the most robust features, the intraclass correlation coefficient (ICC) was computed between the four contours of each lesion obtained with expansion and contraction. This analysis was performed on SUV statistics and texture features and also on their percentage changes. The two-way random effects model (24) was used for ICC calculation. The selection of features least impacted by contouring variability was based on the lower confidence interval of ICC and a threshold of $\geq 0.60$ was used as the cutoff value (25).

The accordance between PET response and MD Anderson criteria was tested with the Fisher exact test.

Both the radiological PET response and the pathological treatment response were logistically modeled. LASSO variable selection was used to identify the variables most capable of classifying response correctly (i.e., Least Absolute Shrinkage and Selection Operator $L_{1}$ penalized regression) (26). A leave-one-out cross-validation (LOOCV) procedure allowed us to fine-tune the LASSO complexity parameter. The choice of LASSO over ridge regression (i.e., $L_{2}$ penalized regression) or elastic-net (i.e. a compromise between the previous two) was motivated by the need to sensibly reduce the number of covariates in the model due to complexity. The ability of SUV statistics and textural features to describe the PET response was first investigated. Then, the possibility of describing the pathological therapy response (MD Anderson index) based on SUV statistics, textural features, and PET response was evaluated. With respect to model calculation, complete response (CR and $\mathrm{pCR}$ ) was considered equal to 1 , while partial response (PR and $\mathrm{PPR}$ ) were considered equal to 0 .

Statistical analyses were carried out with R 4.0.4 (27) and package glmnet 4.1-1 (28) adopted for regularized regression analysis.

\section{RESULTS}

Of the 20 patients selected for the study, three did not have available histology data (one patient refused surgery and two patients were not operated because of systemic disease progression). Two patients did not undergo the second FLT PET/CT scan and were therefore not included in the analysis. Thus, a total of 15 patients were evaluable. Median age at baseline was 54 years with a 10.5 year interquartile range (Table 1). Ten patients underwent image acquisition on the Discovery LS scanner, and the remaining five on the Biograph $20 \mathrm{mCT}$ scanner. Given that lymph nodes were positive in $5 / 15$ patients, only primary lesions were considered for the analysis.

On the basis of MD Anderson criteria, there was a complete absence of neoplastic disease (pCR) classified as RCB-0 in eight 
TABLE 1 | Main patient characteristics.

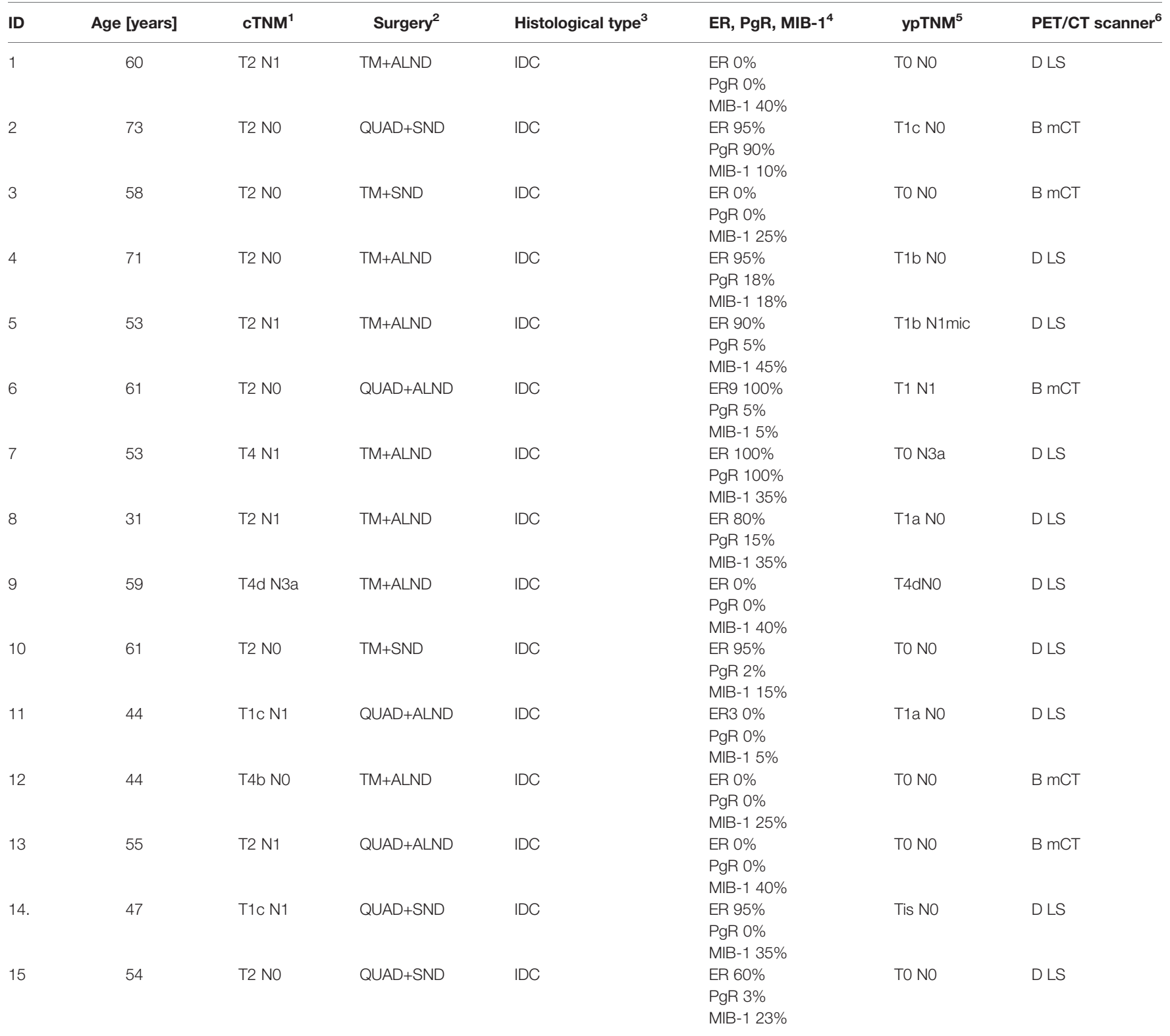

${ }^{1}$ CTNM, cytological TNM classification.

${ }^{2} Q U A D$, quadrantectomy; ALND, axillary lymph node dissection; SND, sentinel node dissection; TM, total mastectomy.

${ }^{3} I D C$, Invasive ductal carcinoma.

${ }^{4} E R$, estrogen receptor; PgR, progesterone receptor; MIB-1, proliferation index.

${ }^{5}$ ypTNM, post-therapy pathological TNM classification.

${ }^{6} D$ LS, Discovery LS PET/CT scanner; B mCT, Biograph 20 mCT PET/CT scanner.

patients and RCB-I in one patient. The remaining six patients showed a partial remission ( $\mathrm{pPR}$ ) classified as RBC-II in five patients and RCB-III in one patient.

On the basis of EORTC 1999 criteria, a comparison of PET images revealed a complete response (CR) to treatment in seven patients and a partial response (PR) in eight patients, with an average reduction in the $S U V_{\max }$ value of $44 \%$.

Comparative analysis of the results showed that PET images were consistent with the subsequent histological examination in 13 (87\%) patients (seven with pCR and six with pPR) (Table 2).
Examples of patients with a PET PR and PET CR to treatment are shown in Figures 2 and 3, respectively.

A discrepancy was observed between PET results and histological findings in two patients (Table 2), both of whom showed a PET PR but a CR at pathological examination (pCR, one RCB-0 and one RCB-I). The Fisher exact test revealed a significant $(\mathrm{p}=0.007)$ association between PET response and MD Anderson criteria.

Among 3 SUV statistics and 148 textural features, all 3 SUV statistics and 39 textural features were classified as robust against 
TABLE 2 | Comparison of the results of PET [EORTC (21)] and MD Anderson [RCB pathological response $(22,23)]$ criteria.

\begin{tabular}{llcr}
\hline & & \multicolumn{2}{c}{ MD criteria } \\
\cline { 3 - 4 } & & pCR & pPR \\
\hline PET response & CR & 7 & 0 \\
& PR & 2 & 6 \\
\hline
\end{tabular}

CR, complete response; PR, partial response.

contour delineation (Table 3). These indices were used for LASSO logistic analysis. The analysis performed on each original data set (FLT1 or FLT 2) showed a lower number of features robust against contour variability (Supplementary Material 3).

Table 4 shows the LOOCV-estimated LASSO logistic model of the binary radiological PET response. In addition to $\mathrm{SUV}_{\max }$, the model selects IVH_VolumeIntFract_90 as an important texture feature for classification. The selected textural feature stands for "Volume at Intensity Fraction 90\%" [IBSI feature classification: BC2M (18)]. This feature belongs to the "Intensity Volume Histogram" feature set [IBSI family classification: P88C (18)] and describes the relationship between discretized intensities and the fraction of volume containing at least one determined intensity value (18). IVH_VolumeIntFract_90 measures the largest volume fraction that has a normalized
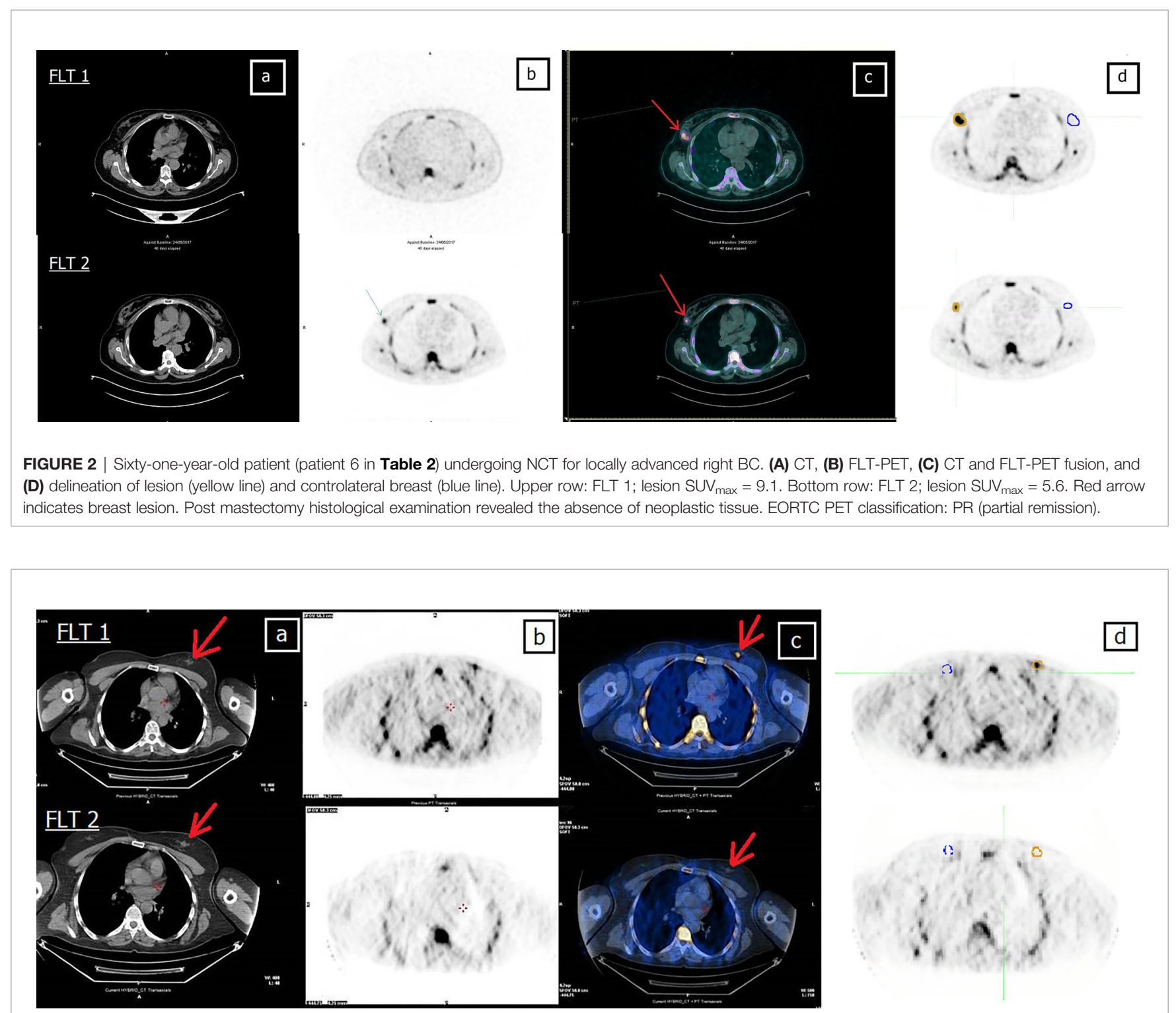

FIGURE 3 | Forty-four-year-old patient (patient 11 in Table 2) undergoing NCT for locally advanced right BC. (A) CT, (B) FLT-PET, (C) CT and FLT-PET fusion, and (D) delineation of lesion (yellow line) and controlateral breast (blue line). Upper row: FLT 1; lesion SUV max $=2.7$. Bottom row: FLT 2; FLT 2 shows the complete disappearance of uptake in the tumor area, still evident at CT (arrow). Red arrow indicates breast lesion. Post mastectomy histological examination revealed the absence of neoplastic tissue. EORTC PET classification: CR (complete remission). 
TABLE 3 | SUV statistics and robustness of textural features robust against contour delineation.

\begin{tabular}{|c|c|c|}
\hline & ICC & ICC lower bound \\
\hline SUV_max & 0.99 & 0.98 \\
\hline SUV_peak & 0.99 & 0.97 \\
\hline SUV_mean & 0.98 & 0.96 \\
\hline ID_Variance & 0.98 & 0.97 \\
\hline ID_Median & 0.94 & 0.87 \\
\hline ID_Min & 0.80 & 0.64 \\
\hline ID_10thPercentile & 0.92 & 0.83 \\
\hline ID_90thPercentile & 0.97 & 0.94 \\
\hline ID_InterquartileRange & 0.89 & 0.78 \\
\hline ID_Range & 0.99 & 0.97 \\
\hline ID_MeanAbsoluteDeviation & 0.96 & 0.92 \\
\hline ID_RobustMeanAbsoluteDeviation & 0.94 & 0.88 \\
\hline ID_MedianAbsoluteDeviation & 0.96 & 0.92 \\
\hline ID_CoefficientOfVariation & 0.94 & 0.88 \\
\hline ID_QuartileCoefficientOfDispersion & 0.81 & 0.64 \\
\hline ID_Energy & 0.97 & 0.92 \\
\hline ID_RootMeanSquare & 0.97 & 0.94 \\
\hline IVH_VolumelntFract_90 & 0.91 & 0.81 \\
\hline IVH_IntensityVolFract_10 & 0.79 & 0.62 \\
\hline IVH_AreaUnderIVHCurve & 0.80 & 0.62 \\
\hline LIF_LocallntensityPeak & 0.98 & 0.96 \\
\hline GLCM_222_1JointAverage & 0.81 & 0.65 \\
\hline GLCM_222_1SumAverage & 0.81 & 0.65 \\
\hline GLCM_222_1AutoCorrelation & 0.81 & 0.64 \\
\hline GLCM_222_1InformationMeasureCor1 & 0.78 & 0.60 \\
\hline GLDZM_GLNonuniformity & 0.85 & 0.71 \\
\hline GLDZM_ZDNonuniformity & 0.82 & 0.65 \\
\hline GLRLM_ShortRunEmphasis & 0.78 & 0.60 \\
\hline GLRLM_LongRunEmphasis & 0.80 & 0.63 \\
\hline GLRLM_HighGLRunEmpha & 0.81 & 0.64 \\
\hline GLRLM_ShortRunHighGLEmpha & 0.80 & 0.63 \\
\hline GLRLM_LongRunHighGLEmpha & 0.83 & 0.67 \\
\hline GLRLM_GLNonuniformity & 0.90 & 0.79 \\
\hline GLRLM_RLNonuniformity & 0.95 & 0.87 \\
\hline GLRLM_RunPercentage & 0.81 & 0.64 \\
\hline GLSZM_LargeZoneHighGLEmpha & 0.90 & 0.80 \\
\hline GLSZM_GLNonuniformity & 0.85 & 0.71 \\
\hline NGLD_HighGLCountEmpha & 0.81 & 0.65 \\
\hline NGLD_HighDepenHighFLEmpha & 0.78 & 0.60 \\
\hline NGLD_GLNonuniformity & 0.89 & 0.78 \\
\hline NGLD_DepCountNonuniformity & 0.84 & 0.69 \\
\hline NID_Coarseness & 0.81 & 0.64 \\
\hline
\end{tabular}

Intraclass correlation coefficient (ICC) calculated using the two-way random effects model. Assumption: absolute agreement. Thresholds: lower bound of $95 \% \mathrm{Cl}$ of ICC and a threshold of 0.60 for ICC $(24,25)$ (good reliability).

intensity of at least $90 \%$ (19). The model showed that a decrease in $\mathrm{SUV}_{\max }$ at FLT2 with respect to baseline was more likely to lead to a CR. Conversely, an increase in IVH_VolumeIntFract_90 led

TABLE 4 | Coefficients, tuning parameters, and classification error rate for the two LASSO logistic models for radiological PET response (26).

\begin{tabular}{lc}
\hline Coefficients & \\
\hline Intercept & -3.316 \\
SUV & \\
IVH_VolumelntFract_90 & 0.041 \\
$\lambda_{\text {min }}$ & -0.005 \\
Classification Error rate & 0.120 \\
\hline
\end{tabular}

The model allows the algorithm to select the covariates to be included in the model without constraints. directly to a higher probability of observing a CR. Additional models are reported in Supplementary Materials 4.

Table 5 shows two LOOCV-estimated LASSO logistic models of the post-surgery binary pathological response (MD Anderson index). The first model was fitted without including PET response as covariate. No linear combination of any subset of the covariates (SUV statistics and texture features) is considered useful for classifying the MD Anderson response. In contrast, the second model was fitted to include the PET response as covariate. The inclusion of PET response among the radiomic covariates (SUV statistics and texture features) resulted in LASSO selecting a combination of variables that were informative about MD Anderson response. Despite the strong association between PET response and MD Anderson index (Table 2), LASSO also selected texture features as informative about MD Anderson classification. The two selected radiomics features are ID_Range and ID_CoefficientOfVariation, which stand for "Intensity-based Range" [IBSI feature classification: 2OJQ (18)] and "Intensitybased Coefficient of Variation" [IBSI feature classification: 7TET (18)], respectively. Both features belong to the "Intensity-based" features set [IBSI family classification: UHIW (18)] which describes how intensity values are distributed within the VOI. In particular, ID_CoefficientOfVariation measures the dispersion of intensity values inside the VOI, while ID_Range is defined as the difference between the maximum and minimum value. The model showed that a decrease in ID_Range or ID_CoefficientOf Variation at FLT2 with respect to the baseline value or a PET response equal to $\mathrm{CR}$ was more likely to lead to a $\mathrm{pCR}$.

\section{DISCUSSION}

The potential value of PET in monitoring response to chemotherapy in breast cancer has yet to be confirmed. The majority of studies to date have been conducted using PET with FDG, while only a few have focused on PET with FLT, mainly in heterogeneous series of patients at different stages of disease, undergoing different chemotherapy regimens and, in particular, with different timing than chemotherapy.

The present study, carried out in a population treated with the same NCT schedule (i.e. six cycles each) showed that an early PET with FLT, performed after the first cycle of therapy, was able

TABLE 5 | Coefficients, tuning parameters, and classification error rate for the two LASSO logistic models for pathological response (MD Anderson criteria) (26).

\begin{tabular}{lcc}
\hline Coefficients & $\begin{array}{c}\text { without } \\
\text { PET response } \\
\text { as covariate }\end{array}$ & $\begin{array}{c}\text { with } \\
\text { PET response } \\
\text { as covariate }\end{array}$ \\
\hline Intercept & 0.405 & -1.842 \\
PET response & - & 1.313 \\
ID_Range & - & 0.006 \\
ID_CoefficientOfVariation & - & 0.001 \\
$\lambda_{\text {min }}$ & 0.332 & 0.204 \\
Classification Error rate & 0.400 & 0.133 \\
\hline
\end{tabular}

The PET response is added to the second model as covariate. Both models allow the algorithm to select the covariates to be included in the model without constraints. 
to classify the pathological response in $100 \%$ of cases. It is worthy of note that, despite the limited number of patients analyzed, treatment modality and schedules were homogeneous.

The use of FLT PET/CT scans in breast cancer management has been described in several studies with small cohorts of patients. In a pilot study of 14 patients, Pio et al. (29) reported that a reduction in mean FLT uptake in primary and metastatic tumors after the first course of chemotherapy was significantly correlated with late tumor marker levels and tumor size. Kenny et al. (30) showed that changes in FLT uptake within the first week of chemotherapy in 13 patients with stage II-IV BC were detectable in FLT images. Their results highlighted that the reduction in SUV uptake observed in 27 lesions on FLT images preceded changes in tumor size and was able to discriminate between clinical response (six patients) and stable disease ( six patients) ( $\mathrm{p}$-value $=0.022$, Mann-Whitney test). In addition, the authors also showed that FLT images could be performed with high reproducibility (repeated FLT image acquisition with a two- to 10-day time interval, $\mathrm{p}$-value $=0.95$ Wilcoxon signed test).

The majority of studies in the literature are based on maximum SUV uptake. Although different statistical indices have been proposed to describe the maximum uptake [e.g. $\left.S U V_{\text {max }}, S_{\text {peak }}, S_{\text {p }} V_{95 \text { th }}(31)\right]$, they are not able to detail the non-uniform uptake distribution of the tracer within the lesions and its variation during therapy. Thus, the information provided by these classic indices may be incomplete. It is well known (10) that lesions can be characterized by heterogeneity of tracer distribution in relation to both cellularity and vascularization, hypoxia, or necrosis. For this reason, the estimate of maximum SUV may not faithfully represent the changes related to the effects of chemotherapy.

Radiomics, the process of computerized extraction of functions from radiographic images, is a new strategy for highlighting subtle changes in the tumor region that works by quantifying the sub-visual patterns that may escape human identification. In a recent review, Sollini et al. (31) evaluated the role of PET radiomics in breast cancer, focusing in particular on methodological aspects. Their analysis highlighted significant heterogeneity in published studies in relation to the acquisition, reconstruction, segmentation, and processing of radiomics, suggesting that much of the current evidence on the clinical role of radiomics is only available at a feasibility level. Textural feature extraction has also been tested on FLT images of other tumor types. Dehdashti et al. (32) analyzed FLT images acquired for 13 patients with advanced colorectal cancer before and 2 weeks after the start of neoadjuvant chemotherapy. The authors showed that, during-therapy, low FLT uptake $\left(\mathrm{SUV}_{\max }<2.2\right)$ and high percentage change in FLT uptake (60\%) were predictive of improved disease-free-survival ( $\mathrm{p}<0.05$ for both values). They also found that pre-therapy FLT uptake was not a significant predictor of outcome and did not correlate with disease-freesurvival. Ulrich et al. (33) exploited the usefulness of radiomics textural feature extraction on FLT images for patients with headand-neck cancer. Thirty patients with advanced-stage oropharyngeal or laryngeal cancer treated with definitive chemoradiotherapy were included in the study. The authors found that smaller and more homogenous lesions (described by different textural feature indices) at baseline were associated with better prognosis ( $\mathrm{p}$-value $<0.05$ ).

In our study, we combined both SUV statistics and radiomic features. The LASSO logistic regression selects the most informative features of the dataset for classification. The usefulness of IVH_VolumeIntFract_90 is, thus, implicit in its selection because otherwise LASSO would have selected only a combination of SUV statistics. Nonetheless, we do not have a separate test set to evaluate the model performance.

Our models show that the information capable of describing the PET response to treatment is localized in the upper part of the SUV histogram, but is not fully reflected by the SUV max. In fact, the models also include the information provided by the textural feature IVH_VolumeIntFract_90. Our findings are in agreement with those of Baiocco et al. (34) who found that the $\mathrm{SUV}_{95 \text { th }}$ (i.e. median computed on the upper $10 \%$ of the SUV distribution) was a more robust index than $\mathrm{SUV}_{\max }$ value for uptake characterization. The present study, and the results reported by Baiocco et al. (34), confirms the role of the upper part of the SUV distribution and highlight the need to define new indices capable of overcoming the limits of classic SUV statistics. In fact, as suggested by Baiocco et al. (34), the single voxel count $\mathrm{SUV}_{\max }$ normally represents an outlier of the SUV histogram.

With regard to the ability to describe the post-surgery pathological response, the LASSO model selected both the PET radiological response and two textural features as most representative of therapy response. The inclusion of two textural features highlights that EORTC criteria alone are inadequate to interpret pathological response and that additional information is needed to correctly asses treatment response based on early imaging. The selection of textural features in both models highlighted the role played by advanced imaging indices in describing the response to treatment. However, the obtained results can only be considered descriptive of the considered patient population, and further investigation in different and larger case series is needed to confirm the predictive power of the model.

Data in the literature have shown that magnetic resonance imaging (MRI), alone or associated with FDG PET, could represent a non-invasive technique for monitoring response to NCT and for assessing residual disease. In particular, in a singlecenter study of 93 patients with breast cancer treated with NCT, Pengel et al. (35) reported that FDG PET and MRI had a complementary predictive ability. Using FDG PET (SUV $V_{\max }$ relative reduction) and MRI (relative change in largest tumor diameter) together in a multivariate analysis combined with breast cancer subtypes, the area under the curve (AUC) was 0.90 (95\% CI: 0.83-0.96) (30). The AUCs of single imaging modality were 0.78 (95\% CI: 0.68-088) for FDG PET and 0.79 (95\% CI: 0.70-0.89) for MRI (36). The association of MRI images and FLT PET/CT may therefore provide further information on response to NCT in breast cancer patients.

A limitation of our study was the intrinsic difference between images in terms of both scanner and parameter acquisition (i.e. 
slice thickness, voxel dimension). It is known that, especially for textural features, these factors may influence the statistical analysis and reduce the robustness of extracted textural feature indices (35). In addition, the starting point of each patient may be different in terms of both maximum uptake and distribution inside the lesion. Given that our clinical interest focused on uptake variation as a surrogate of clinical response and that the absolute value of textural features may be influenced by different scanner acquisition and image characteristics, we chose to use the percentage variation of textural features rather than absolute values. This choice may have overcome the loss in textural feature robustness due to image acquisition parameters. This was also confirmed by the robustness analysis performed on our data, where the data calculated as percentage difference between FLT1 and FLT2 was more robust than the data directly extrapolated from single images (Table 3 and Supplementary Materials 3). A larger patient cohort is needed to properly investigate this point.

Another weakness of our study was the impossibility of evaluating prediction efficacy due to the small sample size involved. In fact, our results, despite their fairly good classification capability, can only be used to describe the current patient set. Indeed, it must be taken into account that the analyzed population was a subgroup of patients extracted from a phase II trial. Thus, a new study with an extended patient cohort would permit the assessment of the prediction capacity of the model in a different population.

\section{CONCLUSIONS}

The reliability of a FLT-PET textural feature approach for the correct and early prediction of response to treatment has yet to be clarified. The choice of the most accurate parameters represents the main problem preventing its routine and generalized use. Our study suggests the potential usefulness of FLT-PET textural feature for early monitoring of NCT response. In particular, the data deriving from radiomics analyses, more informative than those of the semi-quantitative SUV histogram parameters, reinforce the idea that textural feature may be predictive of response to treatment. Further studies on larger populations are warranted to confirm the role of FLT-PET as a

\section{REFERENCES}

1. Bonadonna G, Valagussa P, Brambilla C, Ferrari L, Moliteni A, Terenziani M, et al. Primary Chemotherapy in Operable Breast Cancer: Eight-Year Experience at the Milan Cancer Institute. J Clin Oncol (1998) 16(1):93-100. doi: 10.1200/JCO.1998.16.1.93

2. von Minckwitz G, Raab G, Caputo A, Schütte M, Hilfrich J, Blohmer JU, et al. Doxorubicin With Cyclophosphamide Followed by Docetaxel Every 21 Days Compared With Doxorubicin and Docetaxel Every 14 Days as Preoperative Treatment in Operable Breast Cancer: The GEPARDUO Study of the German Breast Group. J Clin Oncol (2005) 23(12):2676-85. doi: 10.1200/ JCO.2005.05.078

3. Gianni G, Baselga J, Eiermann W, Porta VG, Semiglazov V, Lluch A, et al. Feasibility and Tolerability of Sequential Doxorubicin/Paclitaxel Followed by Cyclophosphamide, Methotrexate, and Fluorouracil and Its Effects on Tumor tool to tailor therapy, reducing the risk of exposing unresponsive patients to unnecessary and harmful cycles of chemotherapy

\section{DATA AVAILABILITY STATEMENT}

The datasets presented in this study can be found in online repositories. The names of the repository/repositories and accession number(s) can be found in the article/Supplementary Material.

\section{ETHICS STATEMENT}

The studies involving human participants were reviewed and approved by Comitato Etico della Romagna, Eudract number: 2014-002602-20; Protocol Code: IRST174.09. The patients/ participants provided their written informed consent to participate in this study.

\section{AUTHOR CONTRIBUTIONS}

Conceptualization: LF, FM, IA, MB, and AS. Methodology: IA, $\mathrm{MB}$, and EL. Formal analysis and investigation: IA, EL, MB, LF, FM, EM, and SA. Writing-original draft preparation: MB, IA, LF, FM, and EL. Writing-review and editing: GP, FM, and SA. Supervision: AS, GP, AF, FM. All authors contributed to the article and approved the submitted version.

\section{ACKNOWLEDGMENTS}

The authors thank Gràinne Tierney for editorial assistance.

\section{SUPPLEMENTARY MATERIAL}

The Supplementary Material for this article can be found online at: https://www.frontiersin.org/articles/10.3389/fonc.2021. 601053/full\#supplementary-material

Response as Preoperative Therapy. Clin Cancer Res (2005) 11(24 Pt 1):871521. doi: 10.1158/1078-0432.CCR-05-0539

4. Esserman LJ, Berry DA, De Michele A, Carey L, Davis SE, Buxton M, et al. Pathologic Complete Response Predicts Recurrence-Free Survival More Effectively by Cancer 828 Eur J Nucl Med Mol Imaging (2015) 42:818-830 Subset: Results From the I-SPY 1 TRIAL-CALGB 150007/ 150012, ACRIN 6657. J Clin Oncol (2012) 30(26):3242-9. doi: 10.1200/ JCO.2011.39.2779

5. Kaufmann M, von Minckwitz G, Mamounas EP, Cameron D, Carey LA, Cristofanilli M, et al. Recommendations From An International Consensus Conference on the Current Status and Future of Neoadjuvant Systemic Therapy in Primary Breast Cancer. Ann Surg Oncol (2012) 19(5):1508-16. doi: 10.1245/s10434-011-2108-2

6. von Minckwitz G, Untch M, Blohmer J, Costa SD, Eidtmann H, Fasching PA, et al. Definition and Impact of Pathologic Complete Response on Prognosis After 
Neoadjuvant Chemotherapy in Various Intrinsic Breast Cancer Subtypes. J Clin Oncol (2012) 30(15):1796-804. doi: 10.1200/JCO.2011.38.8595

7. Eisenhauera EA, Therasseb P, Bogaertsc J, Schwartz LH, Sargent D, Ford R, et al. New Response Evaluation Criteria in Solid Tumours: Revised RECIST Guideline (Version 1.1). Eur J Cancer (2009) 45(2):228-47. doi: 10.1016/ j.ejca.2008.10.026

8. Boellaard R, Delgado-Bolton R, Oyen WJG, Giammarile F, Tatsch K, Eschner W, et al. Fdg PET/CT: EANM Procedure Guidelines for Tumour Imaging: Version 2.0. Eur J Nucl Med Mol Imaging (2015) 42(2):328-54. doi: 10.1007/ s00259-014-2961-x

9. Salskov A, Tammisetti VS, Grierson J, Vasselle H. FLT: Measuring Tumor Cell Proliferation In Vivo With Positron Emission Tomography and 3'Deoxy-3'-18F-Fluorothymidine. Semin Nucl Med (2007) 37(6):429-39. doi: 10.1053/j.semnuclmed.2007.08.001

10. Crippa F, Agresti R, Sandri M, Mariani G, Padovano B, Alessi A, et al. ${ }^{18} \mathrm{f}$-Flt $\mathrm{PET} / \mathrm{CT}$ as An Imaging Tool for Early Prediction of Pathological Response in Patients With Locally Advanced Breast Cancer Treated With Neoadjuvant Chemotherapy: A Pilot Study. Eur J Nucl Med Mol Imaging (2015) 42(6):81830. doi: 10.1007/s00259-015-2995-8

11. Bailly C, Bodet-Milin C, Bourgeois M, Gouard S, Ansquer C, Barbaud M, et al. Exploring Tumor Heterogeneity Using PET Imaging: The Big Picture. Cancers (2019) 11(9):1282. doi: 10.3390/cancers11091282

12. Jamal-Hanjani M, Quezada SA, Larkin J, Swanton C. Translational Implications of Tumor Heterogeneity. Clin Cancer Res (2015) 21(6):125866. doi: 10.1158/1078-0432.CCR-14-1429

13. El Naqa I, Grigsby P, Apte A, Kidd E, Donnelly E, Khullar D, et al. Exploring Feature-Based Approaches in PET Images for Predicting Cancer Treatment Outcomes. Pattern Recogn (2009) 42(6):1162-71. doi: 10.1016/ j.patcog.2008.08.011

14. Cook GJ, O’Brien ME, Siddique M, Chicklore S, Loi HY, Sharma B, et al. NonSmall Cell Lung Cancer Treated With Erlotinib: Heterogeneity of ${ }^{18}$ F-FDG Uptake at PET-Association With Treatment Response and Prognosis. Radiology (2015) 276(3):883-93. doi: 10.1148/radiol.2015141309

15. Lee HS, Oh JS, Park YS, Jang SJ, Choi IS, Ryu J-S, et al. Differentiating the Grades of Thymic Epithelial Tumor Malignancy Using Textural Features of Intratumoral Heterogeneity Via ${ }^{18}$ F-FDG Pet/Ct. Ann Nucl Med (2016) 30 (4):309-19. doi: 10.1007/s12149-016-1062-2

16. Hyun SH, Kim HS, Choi SH, Choi DW, Lee JK, Lee KH, et al. Intratumoral Heterogeneity of ${ }^{18}$ F-FDG Uptake Predicts Survival in Patients With Pancreatic Ductal Adenocarcinoma. Eur J Nucl Med Mol Imaging (2016) 43 (8):1461-8. doi: 10.1007/s00259-016-3316-6

17. Rausch I, Cal-González J, Dapra D, Gallowitsch HJ, Lind P, Beyer T, et al. Performance Evaluation of the Biograph Mct Flow PET/CT System According to the NEMA Nu2-2012 Standards. EJNMMI Phys (2015) 2:26. doi: 10.1186/ s40658-015-0132-1

18. Bettinelli A, Branchini M, De Monte F, Scaggion A, Paiusco M. Technical Note: An IBEX Adaption Toward Image Biomarker Standardization. Med Phys (2020) 47(3):1167-73. doi: 10.1002/mp.13956

19. Zwanenburg A, Leger S, Vallières M, Löck S. Image Biomarker Standardisation Initiative (2016). Available at: https://www.arxiv.org.

20. Zwanenburg A, Leger S, Vallières M, Abdalah MA, Aerts HJWL, Apte A, et al. The Image Biomarker Standardization Initiative: Standardized Quantitative Radiomics for High-Throughput Image-Based Phenotyping. Radiology (2020) 295(2):328-38. doi: 10.1148/radiol.2020191145

21. Young H, Baum R, Cremerius U, Herholz K, Hoekstra O, Lammertsma AA, et al. Measurement of Clinical and Subclinical Tumor Response Using $\left[{ }^{18} \mathrm{~F}\right]$ Were Used. Fluorodeoxyglucose and Positron Emission Tomography: Review and 1999 EORTC Recommendations. European Organization for Research and Treatment of Cancer (Eortc) PET Study Group. Eur J Cancer (1999) 35:1773-82. doi: 10.1016/S0959-8049(99)00229-4

22. Wolff AC, Hammond MEH, Hicks DG, Dowsett M, McShane LM, Allison $\mathrm{KH}$, et al. Recommendations for Human Epidermal Growth Factor Receptor 2 Testing in Breast Cancer. American Society of Clinical Oncology/College of
American Pathologists Clinical Practice Guideline Update. J Clin Oncol (2013) 31(31):3997-4013. doi: 10.1200/JCO.2013.50.9984

23. The University of Texas Md Anderson Cancer Center Residual Cancer Burden Calculator. Available at: http://www3.mdanderson.org/app/medcalc/index. cfm?pagename=jsconvert3 (Accessed 29 Jan 2015).

24. McGraw K, Wong SP. Forming Inferences About Some Intraclass Correlation Coefficients. Psychol Methods (1996) 1(1):30-46. doi: 10.1037/1082-989X.1.1.30

25. Landis JR, Koch GG. The Measurement of Observer Agreement for Categorical Data. Biometrics (1977) 33(1):159-74. doi: 10.2307/2529310

26. Hastie T, Tibshirani R, Friedman J. The Elements of Statistical Learning: Data Mining, Inference, and Prediction. Berlin, Germany: Springer Science \& Business Media (2009).

27. Core R, Team R. A Language and Environment for Statistical Computing. Vienna, Austria: R Foundation for Statistical Computing (2019). Available at: https://www.R-project.org/.

28. Friedman J, Hastie T, Tibshirani R. Regularization Paths for Generalized Linear Models Via Coordinate Descent. J Stat Softw (2010) 33(1):1-22. doi: 10.18637/jss.v033.i01

29. Pio BS, Park CK, Pietras R, Hsueh W-A, Satyamurthy N, Pegram MD, et al. Usefulness of 3'-[F-18]Fluoro-3'-deoxythymidine With Positron Emission Tomography in Predicting Breast Cancer Response to Therapy. Mol Imaging Biol (2006) 8(1):36-42. doi: 10.1007/s11307-005-0029-9

30. Kenny L, Coombes RC, Vigushin DM, Al-Nahhas A, Shousha S, Aboagye EO, et al. Imaging Early Changes in Proliferation at 1 Week Post Chemotherapy: A Pilot Study in Breast Cancer Patients With 3'-Deoxy-3'-[18F] Fluorothymidine Positron Emission Tomography. Eur J Nucl Med Mol Imaging (2007) 34(9):1339-47. doi: 10.1007/s00259-007-0379-4

31. Sollini M, Cozzi L, Ninatti G, Antunovic L, Cavinato L, Chiti A, et al. PET/CT Radiomics in Breast Cancer: Mind the Step. Methods (2020) S1046-2023 (19):30263-4. doi: 10.1016/j.ymeth.2020.01.007

32. Dehdashti F, Grigsby PW, Myerson RJ, Nalbantoglu I, Ma C, Siegel BA. Positron Emission Tomography With [(18)F]-3'-deoxy- 3'Fluorothymidine (FLT) as a Predictor of Outcome in Patients With Locally Advanced Resectable Rectal Cancer: A Pilot Study. Mol Imaging Biol (2013) 15 (1):106-13. doi: 10.1007/s11307-012-0566-y

33. Ulrich EJ, Menda Y, Boles Ponto LL, Anderson CM, Smith BJ, Sunderland JJ, et al. Flt PET Radiomics for Response Prediction to Chemoradiation Therapy in Head and Neck Squamous Cell Cancer. Tomography (2019) 5(1):161-9. doi: $10.18383 /$ j.tom. 2018.00038

34. Baiocco S, Matteucci F, Mezzenga E, Caroli P, Di Iorio V, Cittanti C, et al. $\mathrm{SUV}_{95 \text { th }}$ as a Reliable Alternative to $\mathrm{SUV}_{\text {max }}$ for Determining Renal Uptake in $\left[{ }^{68} \mathrm{Ga}\right]$ PSMA Pet/Ct. Mol Imaging Biol (2020) 22(4):1070-7. doi: 10.1007/ s11307-019-01451-1

35. Pengel KE, Koolen BB, Loo CE, Vogel WV, Wesseling J, Lips EH, et al. Combined Use of ${ }^{18}$ F-FDG PET/CT and MRI for Response Monitoring of Breast Cancer During Neoadjuvant Chemotherapy. Eur J Nucl Med Mol Imaging (2014) 41:1515-24. doi: 10.1007/s00259-014-2770-2

36. Shiri I, Rahmim A, Ghaffarian P, Geramifar P, Abdollahi H, Bitarafan-Rajabi A. The Impact of Image Reconstruction Settings on ${ }^{18}$ F-FDG PET Radiomic Features: Multi-Scanner Phantom and Patient Studies. Eur Rad (2017) 27:4498-509. doi: 10.1007/s00330-017-4859-z

Conflict of Interest: The authors declare that the research was conducted in the absence of any commercial or financial relationships that could be construed as a potential conflict of interest.

Copyright (๑ 2021 Fantini, Belli, Azzali, Loi, Bettinelli, Feliciani, Mezzenga, Fedeli, Asioli, Paganelli, Sarnelli and Matteucci. This is an open-access article distributed under the terms of the Creative Commons Attribution License (CC BY). The use, distribution or reproduction in other forums is permitted, provided the original author(s) and the copyright owner(s) are credited and that the original publication in this journal is cited, in accordance with accepted academic practice. No use, distribution or reproduction is permitted which does not comply with these terms. 